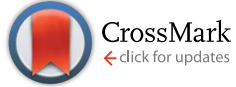

Cite this: Soft Matter, 2015, 11, 3062

\title{
Glassy dynamics of poly(2-vinyl-pyridine) brushes with varying grafting density $\dagger$
}

\author{
Nils Neubauer, ${ }^{* a}$ René Winkler, ${ }^{\mathrm{b}}$ Martin Tress, ${ }^{a}$ Petra Uhlmann, ${ }^{\mathrm{b}}$ Martin Reiche, ${ }^{\mathrm{c}}$ \\ Wycliffe Kiprop Kipnusu ${ }^{a}$ and Friedrich Kremer $^{a}$
}

The molecular dynamics of poly(2-vinyl-pyridine) (P2VP) brushes is measured by Broadband Dielectric Spectroscopy (BDS) in a wide temperature $(250 \mathrm{~K}$ to $440 \mathrm{~K})$ and broad spectral $(0.1 \mathrm{~Hz}$ to $1 \mathrm{MHz})$ range. This is realized using nanostructured, highly conductive silicon electrodes being separated by silica spacers as small as $35 \mathrm{~nm}$. A "grafting-to"-method is applied to prepare the P2VP-brushes with five different grafting densities $\left(0.030 \mathrm{~nm}^{-2}\right.$ to $\left.0.117 \mathrm{~nm}^{-2}\right)$, covering the "true-brush" regime with highly stretched coils and the "mushroom-to-brush" transition regime. The film thickness ranges between 1.8 to $7.1( \pm 0.2) \mathrm{nm}$. Two relaxations are observed, an Arrhenius-like process being attributed to fluctuations in the poly(glycidyl-methacrylate) (PGMA) linker used for the grafting reaction and the segmental dynamics (dynamic glass transition) of the P2VP brushes. The latter is characterized by a Vogel-FulcherTammann dependence similar to bulk P2VP. The results can be comprehended considering the length scale on which the dynamic glass transition ( $\leq 1 \mathrm{~nm}$ ) takes place.

Received 26th January 2015

Accepted 25th February 2015

DOI: $10.1039 / c 5 s m 00213 c$

www.rsc.org/softmatter

\section{Introduction}

Polymer brushes are subject to intensive research with a broad range of interests. Surface grafted polymer layers are on the one hand an interesting field from a theoretical point of view, as they represent a model system for macromolecules in confined geometry. ${ }^{\mathbf{1 , 2}}$ In recent years, much effort has been made to develop and improve superior synthesis strategies, ${ }^{3-6}$ getting tethered polymer layers with high grafting densities and specific molecular architecture for different materials and material combinations. Depending on the specific purpose, systems like mixed brushes, consisting of two polymer components, nanoparticle-brush composites or brushes controlling cell adhesion have been established. The use of stimuli responsive polymer brushes allows switching and fine-tuning of surface properties., ${ }^{3,7-11}$

For polymer layers of only a few nanometers thickness, about two decades of controversial discussion focussed the question whether or not glassy dynamics and glass transition deviate from the well-known bulk properties. ${ }^{12,13}$ Interfacial interactions between the polymer and its confining surfaces were assumed to induce different dynamics. ${ }^{\mathbf{1 4}-16}$ However, recent results coincidently indicate that glassy dynamics of thin polymer layers ${ }^{\mathbf{1 3 , 1 7 - 2 6}}$ and even isolated polymer chains ${ }^{27}$ is

${ }^{a}$ Institute of Experimental Physics I, Leipzig University, 04103 Leipzig, Germany. E-mail: nils.neubauer@uni-leipzig.de

${ }^{b}$ Leibniz-Institut für Polymerforschung Dresden e.V., 01069 Dresden, Germany ${ }^{c}$ Max Planck Institute of Microstructure Physics, 06120 Halle (Saale), Germany

$\dagger$ Electronic supplementary information (ESI) available: See DOI: $10.1039 / \mathrm{c} 5 \mathrm{sm} 00213 \mathrm{c}$ identical to bulk dynamics. In contrast, the calorimetric glass transition may deviate from the bulk value which is, according to recently developed theories, rather related to the finite system size $^{21,28-32}$ or adsorption. ${ }^{26,33}$ Compared to these deposited layers, chemical bonding of polymers to the substrate represents a much stronger attractive interaction. In the case of poly(methyl methacrylate), an increase of the calorimetric glass transition has been found for grafted chains while spin cast layers did not show such changes although the substrate varied from hydrophilic to strongly hydrophobic. ${ }^{34}$ Since the calorimetric glass transition is no reliable measure of segmental dynamics, ${ }^{21}$ the present work employs Broadband Dielectric Spectroscopy (BDS) as a technique directly accessing this process. ${ }^{35}$ In this study, the molecular dynamics of poly(2-vinyl-pyridine) (P2VP) polymer brushes is investigated by means of BDS. Since each chain segment carries a dipole moment, it allows for the measurement of the segmental mode, or $\alpha$-relaxation, of the polymer, which is the structural relaxation underlying the dynamic glass transition. Dielectric measurements down to the level of a few-nm thin films are possible with a recently developed nanostructured electrode arrangement. ${ }^{36}$ Silica pistons with a height of a few tens of nm serve as spacers to guarantee a small and welldefined distance between the electrodes.

\section{Experimental}

The electrodes of the sample capacitor consist of two highly conductive silicon wafers with a specific resistance $\rho<0.01 \Omega \mathrm{cm}$ purchased from Si-Mat and a $200 \mathrm{~nm}$-thick Al-layer deposited 


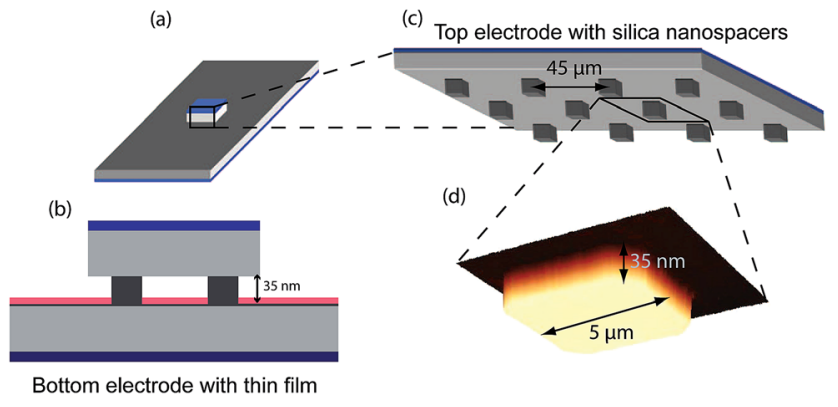

Fig. 1 Sample arrangement: the capacitor (a) is composed of two highly doped silicon electrodes $(\rho<0.01 \Omega \mathrm{cm}$, bottom electrode: $10 \times$ $20 \mathrm{~mm}^{2}$, top electrode: $1 \times 1 \mathrm{~mm}^{2}$; (b) cross section of the sample capacitor). The top electrode is covered by an array of insulating silica nanostructures, serving as spacers (c). The spacers have a height of 35 $\mathrm{nm}$, as confirmed by AFM scans (d), and enable one for dielectric measurements without evaporating a metal counter electrode.

on the backside. The lower electrode with a native oxide layer of about 1-2 $\mathrm{nm}$ is used as substrate for the polymer brush preparation. After cutting the wafer in $10 \times 20 \mathrm{~mm}^{2}$ pieces the wafer dice are cleaned in an ultrasonic bath with ethanol followed by oxygen plasma treatment. To graft the P2VP chains on the substrate, a two-step "grafting-to" procedure is applied., First, a thin layer $(d \approx 2.5 \mathrm{~nm}$ ) of poly(glycidyl-methacrylate)
(PGMA) $\left(M_{\mathrm{n}}=17.5 \mathrm{~kg} \mathrm{~mol}{ }^{-1}, M_{\mathrm{w}}=29.7 \mathrm{~kg} \mathrm{~mol}^{-1}\right.$, obtained from Polymer Source Inc.) is deposited from a $0.02 \mathrm{wt} \%$ chloroform solution and annealed at $100{ }^{\circ} \mathrm{C}$ for $20 \mathrm{~min}$. The PGMA serves as an anchoring layer. In a second step, carboxyl terminated poly(2-vinyl-pyridine) (P2VP-COOH, $M_{\mathrm{n}}=40.6 \mathrm{~kg} \mathrm{~mol}^{-1}$, $M_{\mathrm{w}}=43.8 \mathrm{~kg} \mathrm{~mol}{ }^{-1}$, obtained from Polymer Source, Inc.) is spin-coated from a 1 wt $\%$ THF solution. Subsequently, by applying annealing times from $1 \mathrm{~min}$ to $16 \mathrm{~h}$, the grafting process of the $\mathrm{P} 2 \mathrm{VP}-\mathrm{COOH}$ chains is interrupted at different times. As a result, samples with different grafting densities are prepared. Finally, the samples are rinsed in THF to remove ungrafted polymer chains.

As top electrode a $1 \times 1 \mathrm{~mm}^{2}$ silicon wafer dice (Fig. 1) is used, which is accomplished with an array of highly insulating silica spacers having a quadratic cross section of $5 \times 5 \mu \mathrm{m}^{2}$ and a height of $35 \mathrm{~nm}$. In order to clean the dice, acetone rinsing and plasma treatment followed by snow jet blasting are applied.

To check the quality and homogeneity of the prepared polymer brushes Atomic Force Microscopy (AFM) is carried out employing a Vecco Dimension 3000 AFM in tapping mode. Film thicknesses are determined by ellipsometry (Sentech SE-402 scanning microfocus ellipsometer).

For the BDS measurements a Novocontrol Alpha analyzer (spectral range from $1 \mathrm{mHz}$ to $10 \mathrm{MHz}$ ) was used, in combination with a Quatro temperature controller to cover a temperature range of $250 \mathrm{~K}$ to $440 \mathrm{~K}$ with a relative stabilization accuracy

Table 1 Sample details: P2VP brushes with five different grafting densities are prepared by applying five different annealing times for the P2VP$\mathrm{COOH}$ chains on top of a PGMA layer. The PGMA and P2VP layer thicknesses $d_{\mathrm{PGMA}}$ and $d_{\mathrm{P} 2 \mathrm{VP}}$ respectively are measured by ellipsometry. The corresponding grafting density $\sigma_{\mathrm{P} 2 \mathrm{VP}}$ and the reduced grafting density $\Sigma$ can be calculated. Samples $1-3$ are clearly in the "true brush" regime, while samples 4 and 5 cover the "mushroom-to-brush" transition regime

\begin{tabular}{|c|c|c|c|c|c|}
\hline Sample & Annealing time & $d_{\mathrm{PGMA}}[\mathrm{nm}]$ & $d_{\mathrm{P} 2 \mathrm{VP}}[\mathrm{nm}]$ & $\sigma_{\mathrm{P} 2 \mathrm{VP}}\left[\mathrm{nm}^{-2}\right]\left( \pm 0.005 \mathrm{~nm}^{-2}\right)$ & $\Sigma$ \\
\hline 1 & $16 \mathrm{~h}$ & $2.5 \pm 0.1$ & $7.1 \pm 0.2$ & 0.117 & $9 \pm 2$ \\
\hline 2 & $30 \mathrm{~min}$ & $2.4 \pm 0.1$ & $6.4 \pm 0.1$ & 0.106 & $8 \pm 2$ \\
\hline 4 & $6 \min$ & $2.4 \pm 0.2$ & $4.5 \pm 0.2$ & 0.074 & $6 \pm 1.5$ \\
\hline 5 & $1 \mathrm{~min}$ & $2.6 \pm 0.1$ & $1.8 \pm 0.1$ & 0.030 & $2.4 \pm 1$ \\
\hline
\end{tabular}
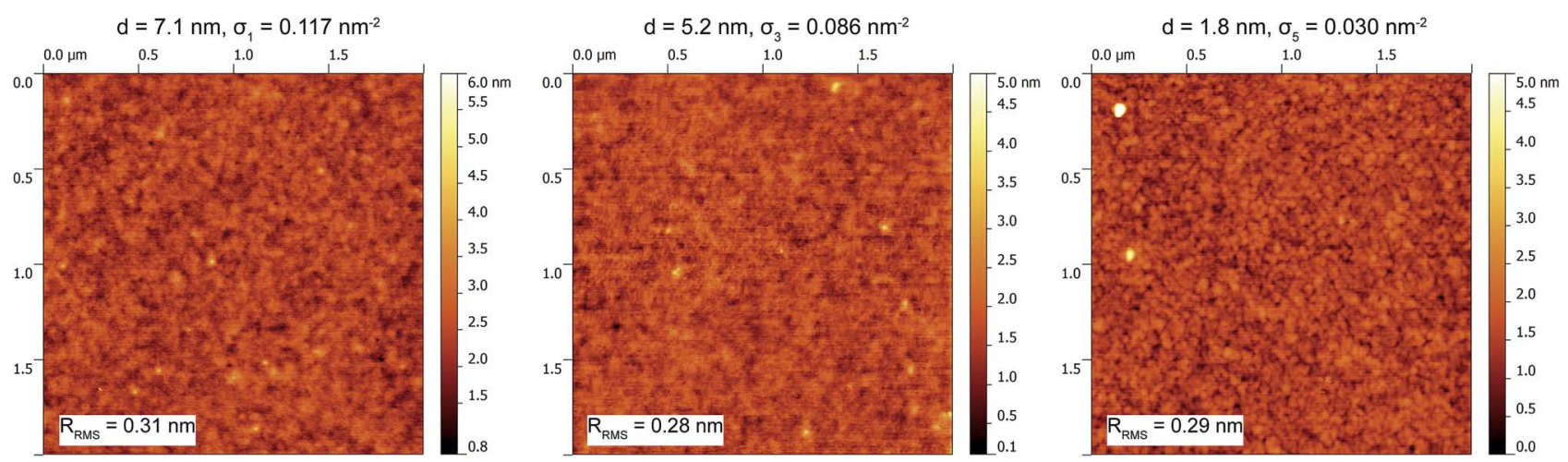

Fig. 2 AFM scans providing the quality of the prepared P2VP brushes with varying grafting density. Three example images are shown for three different grafting densities. The scans reveal homogeneous films after the grafting procedure with a roughness of about $R_{\mathrm{RMS}} \approx 0.3 \mathrm{~nm}$ for all five grafting densities. Film thicknesses, as specified on the top of each image, were determined by ellipsometry ranging from $7.1 \mathrm{~nm}$ to $1.8 \mathrm{~nm}$ $( \pm 0.2 \mathrm{~nm})$. 
of $0.1 \mathrm{~K}$. The measurement accuracy of the dielectric spectrometers is $10^{-4}$. Due to uncertainties in the control of the separation between the solid surfaces of the nanostructured electrodes, the dielectric strength of the relaxation processes can be only determined with an absolute accuracy of $\approx 30 \%$. This does of course not influence the precision with which the maximum position of the relaxation processes and hence the mean relaxation rate can be deduced.

\section{Results and discussion}

After each step of the polymer brush preparation, the layer thickness was measured by ellipsometry. The measured layer thicknesses for PGMA and P2VP for the different annealing times are listed in Table 1 . Using $\sigma=h \rho N_{\mathrm{A}} / M_{\mathrm{n}}$, the corresponding grafting densities, ranging from $0.030 \mathrm{~nm}^{-2}$ to $0.117 \mathrm{~nm}^{-2}$, can be calculated ${ }^{3}$ (Table 1 ). With the radius of gyration of the P2VP coils $\left(R_{\mathrm{g}} \approx 5 \mathrm{~nm}\right)$ the reduced grafting density ${ }^{6}$ is given by $\Sigma=$ $\pi R_{\mathrm{g}}{ }^{2} \sigma$. Using the classification of Brittain et al. ${ }^{6}$ the samples cover the "true-brush" regime with highly stretched coils and the "mushroom-to-brush" transition regime.

The film quality of the prepared P2VP polymer brushes is also checked by AFM measurements. Typical images of three grafting densities are given in Fig. 2 and show a homogeneous surface with a typical roughness in the order of $R_{\mathrm{RMS}} \approx 0.3 \mathrm{~nm}$. This confirms, together with the ellipsometric data, the homogeneity of the prepared films of P2VP brushes.

To access the molecular dynamics of the prepared P2VP brushes, BDS measurements were carried out. Fluctuations of dipolar segments of the polymer chains are probed by measuring the complex dielectric function $\varepsilon^{*}=\varepsilon^{\prime}-\mathrm{i} \varepsilon^{\prime \prime}$ with the real $\left(\varepsilon^{\prime}\right)$ and the imaginary or loss $\left(\varepsilon^{\prime \prime}\right)$ parts. Measurements were performed in the frequency range from $10^{-1} \mathrm{~Hz}$ to $10^{6} \mathrm{~Hz}$ at temperatures ranging from $250 \mathrm{~K}$ to $440 \mathrm{~K}$. Fig. 3 shows the dielectric loss $\varepsilon^{\prime \prime}$ spectra of the P2VP brushes at three different temperatures ( $400 \mathrm{~K}$ to $420 \mathrm{~K}$ ). Several features can be observed in the spectra. Beside the molecular relaxations of PGMA and P2VP the specific structure of the sample capacitor with silicon wafer electrodes, an interfacial silica layer and the silica spacers with their respective dielectric properties contribute to the measured signal. To account for the multiple components, an equivalent circuit model is used to describe the spectra (see ESI $\dagger$ ). Each component is included with a certain thickness and a complex dielectric function. With this model a fit function for the measured dielectric loss is deduced (solid lines in the spectra of Fig. 3) and can be used to extract the net dielectric properties of the components, e.g. the P2VP-brushes. Two linear contributions at the low and high frequency side are present which originate from a parasitic conductivity (related to the silica spacers or contaminating particles between the electrodes) and the limited resistivity of the silicon electrodes, respectively. A conductivity part of the thin P2VP layer leads to a polarization peak, observable in a frequency range of $10^{-1} \mathrm{~Hz}$ to $10^{2} \mathrm{~Hz}$ in the plotted temperature range of $400 \mathrm{~K}$ to $420 \mathrm{~K}$. The remaining molecular relaxations of PGMA and P2VP are modeled by two Havriliak-Negami-functions $\varepsilon^{*}=\varepsilon_{\infty}+\Delta \varepsilon /(1+$ $\left.\left(\mathrm{i} \omega \tau_{\mathrm{HN}}\right)^{\beta}\right)^{\gamma}$, an empirical function to describe relaxation processes. Reference measurements of a PGMA layer with (2.5 \pm 0.1) $\mathrm{nm}$ thickness were done to determine the PGMA contribution and show a process in the temperature range of $250 \mathrm{~K}$ to $430 \mathrm{~K}$ (see ESI $\dagger$ ). In Fig. 3 this PGMA-relaxation is observed in the frequency range $10^{5} \mathrm{~Hz}$ to $10^{6} \mathrm{~Hz}$. Finally, the process lying within $10^{2} \mathrm{~Hz}$ to $10^{5} \mathrm{~Hz}$ is assigned to the P2VP $\alpha$-relaxation, i.e. the dynamic glass transition. With decreasing grafting density, the intensity of the $\alpha$-relaxation peak decreases due to the reduced layer thickness. In the present measurement configuration with $35 \mathrm{~nm}$ silica spacers and with the present bilayer

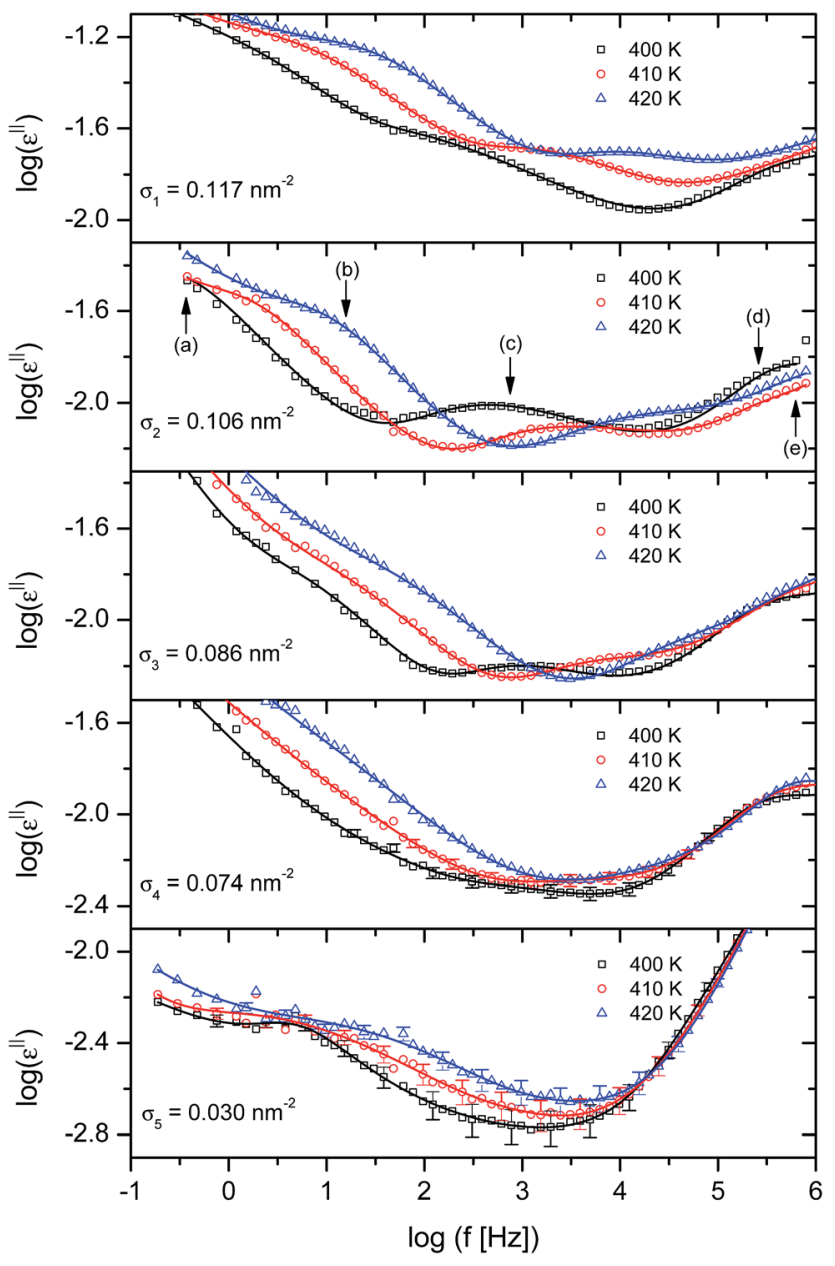

Fig. 3 Dielectric loss $\varepsilon^{\prime \prime}$ spectra of P2VP brushes on a PGMA anchoring layer for five different grafting densities at varying temperatures as indicated. Several contributions are observed and marked by arrows in the spectra of grafting density $\sigma_{2}$ : (a) a linear contribution due to a parasitic conductivity of the spacers or contaminating particles between the electrodes, (b) a polarization which originates from the conductivity of P2VP, (c) the $\alpha$-relaxation of P2VP, (d) a PGMA-relaxation, (e) and a linear contribution due to the limited conductivity of the silicon electrodes. The fit function (solid lines) is deduced from an equivalent circuit model (see ESI†), which includes complex dielectric functions of the multiple components present in the sample capacitor. Thereby, the molecular relaxations of P2VP and PGMA are modelled by two Havriliak-Negami functions. The relative experimental accuracy is given by the size of the symbols if not indicated otherwise. The absolute accuracy of the relaxation strength is estimated to be $\pm 30 \%$. 


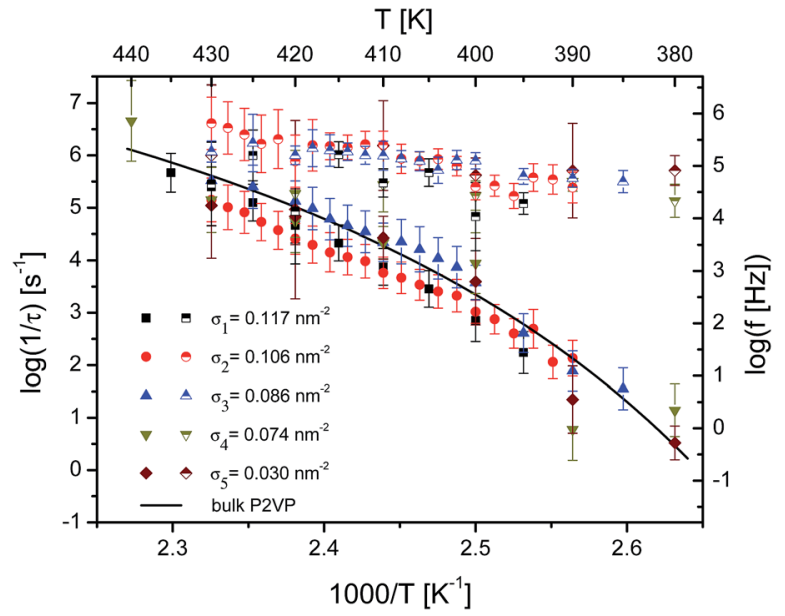

Fig. 4 Activation plot of the P2VP $\alpha$-relaxation (filled symbols) and the PGMA-relaxation (half-filled symbols) of P2VP brushes on a PGMA layer for five different grafting densities as indicated. For all grafting densities the PGMA relaxation has an Arrhenius-like thermal activation while the P2VP-brushes follow a Vogel-Fulcher-Tammann temperature dependence. The solid line shows the mean relaxation rates of bulk P2VP. The error bars show the accuracy for determining the mean relaxation rate.

preparation technique, the two lowest grafting densities $\left(\sigma_{4}, \sigma_{5}\right.$ in Fig. 3) already represent the limit of sensitivity for getting signals of the P2VP $\alpha$-relaxation. The temperature dependence of the maximum position of the $\alpha$-relaxation is depicted in the activation plot (Fig. 4). It shows a Vogel-Fulcher-Tammann temperature dependence for all the measured grafting densities, a characteristic feature of the dynamic glass transition. Furthermore, within the experimental uncertainty, no deviations from bulk dynamics are observable. Hence, the conformational differences in the varying brush stages have no influence on the glassy dynamics.

Similar results were reported by Mapesa et al. ${ }^{20}$ for thin layers of polyisoprene spincast onto substrates. Their work shows that the segmental dynamics remains identical while the chain conformation is subject of pronounced alterations due to changes in the layer thickness.

The fact that the mean relaxation rate of the segmental dynamics is independent of the grafting density shows that the potential between the chain segments is identical for all samples. On the one hand, this is remarkable according to the fact that the coiled polymer chains in such brushes are expected to stretch in perpendicular to the substrate and, by that, take an entropic force. ${ }^{1,2}$ As the results show, the impact of such effects on the intermolecular potential can be neglected. On the other hand, our finding can be to some extent anticipated from the work of Tress et $a .^{27}$ who found bulk-like glassy dynamics in condensed isolated P2VP polymer chains. In their case, isolated P2VP chains were, in very flat (height $\approx 3-6 \mathrm{~nm}$ ) conformation, subject to attractive interactions with terminal hydroxyl groups of a silica surface which had no impact on the glassy dynamics of most of the segments. These results indicate a bulk-like density in these systems.

\section{Conclusion}

Broadband dielectric measurements were carried out to reveal the molecular dynamics of P2VP brushes with different grafting densities ranging from highly stretched brushes to the mushroom-to-brush transition regime. The quality of the prepared thin films was checked by AFM and ellipsometry. Using nanostructured electrodes with $35 \mathrm{~nm}$ silica spacers it was possible to measure the dynamic glass transition of the P2VP brushes for all the prepared grafting densities. The finding that the mean relaxation rate of the P2VP brushes coincides with that of the bulk demonstrates that the intermolecular potentials for segmental fluctuations are similar. This indicates that also the density is not altered, a proposition, which to the best of our knowledge has not yet been proven experimentally, but being commonly anticipated in the determination of the grafting density from the layer thickness. Hence, the presented results corroborate the assumption of bulk density in brush systems in the examined range of grafting densities.

\section{Acknowledgements}

We like to thank Carolin Böhm and Wiktor Skokow for the technical support. Furthermore, we gratefully acknowledge financial support from DFG within SFB TRR102 and Leipzig School of Natural Sciences, "Building with Molecules and NanoObjects" (BuildMoNa).

\section{References}

1 S. T. Milner, Science, 1991, 251, 905-914.

2 P. G. de Gennes, Macromolecules, 1980, 13, 1069-1075.

3 R. C. Advincula, W. J. Brittain, K. C. Caster and J. Rühe, Polymer Brushes: Synthesis, Characterization, Applications, Wiley-VCH Verlag GmbH \& Co. KGaA, Weinheim, 2004.

4 M. Tirrell, S. Patel and G. Hadziioannou, Proc. Natl. Acad. Sci. U. S. A., 1987, 84, 4725-4728.

5 B. Zhao and W. J. Brittain, Prog. Polym. Sci., 2000, 25, 677710.

6 W. J. Brittain and S. Minko, J. Polym. Sci., Part A: Polym. Chem., 2007, 45, 3505-3512.

7 L. Ionov, A. Sidorenko, M. Stamm, S. Minko, B. Zdyrko, V. Klep and I. Luzinov, Macromolecules, 2004, 37, 7421-7423.

8 S. Gupta, P. Uhlmann, M. Agrawal, S. Chapuis, U. Oertel and M. Stamm, Macromolecules, 2008, 41, 2874-2879.

9 I. Tokareva, S. Minko, J. H. Fendler and E. Hutter, J. Am. Chem. Soc., 2004, 126, 15950-15951.

10 O. Azzaroni, J. Polym. Sci., Part A: Polym. Chem., 2012, 50, 3225-3258.

11 P. Uhlmann, H. Merlitz, J.-U. Sommer and M. Stamm, Macromol. Rapid Commun., 2009, 30, 732-740.

12 F. Kremer, E. U. Mapesa, M. Tress and M. Reiche, Recent Advances in Broadband Dielectric Spectroscopy, Springer, Netherlands, 2013, ch. 12, pp. 163-178.

13 F. Kremer, M. Tress and E. U. Mapesa, J. Non-Cryst. Solids, 2015, 407, 277-283. 
14 J. N. D'Amour, U. Okoroanyanwu and C. W. Frank, Microelectron. Eng., 2004, 73-74, 209-217.

15 C. Zhang, Y. Fujii and K. Tanaka, ACS Macro Lett., 2012, 1, 1317-1320.

16 S. Gao, Y. P. Koh and S. L. Simon, Macromolecules, 2013, 46, 562-570.

17 M. Tress, M. Erber, E. U. Mapesa, H. Huth, J. Muller, A. Serghei, C. Schick, K.-J. Eichhorn, B. Voit and F. Kremer, Macromolecules, 2010, 43, 9937-9944.

18 E. U. Mapesa, M. Erber, M. Tress, K. J. Eichhorn, A. Serghei, B. Voit and F. Kremer, Eur. Phys. J.: Spec. Top., 2010, 189, 173-180.

19 E. U. Mapesa, M. Tarnacka, E. Kaminska, K. Adrjanowicz, M. Dulski, W. Kossack, M. Tress, W. K. Kipnusu, K. Kaminski and F. Kremer, RSC Adv., 2014, 4, 28432-28438.

20 E. U. Mapesa, M. Tress, G. Schulz, H. Huth, C. Schick, M. Reiche and F. Kremer, Soft Matter, 2013, 9, 10592-10598.

21 V. M. Boucher, D. Cangialosi, H. Yin, A. Schönhals, A. Alegria and J. Colmenero, Soft Matter, 2012, 8, 5119-5122.

22 K. Paeng, S. F. Swallen and M. D. Ediger, J. Am. Chem. Soc., 2011, 133, 8444-8447.

23 K. Paeng, R. Richert and M. D. Ediger, Soft Matter, 2012, 8, 819-826.

24 K. Paeng and M. D. Ediger, Macromolecules, 2011, 44, 70347042.

25 A. Serghei, H. Huth, C. Schick and F. Kremer, Macromolecules, 2008, 41, 3636-3639.

26 S. Napolitano and M. Wübbenhorst, Nat. Commun., 2011, 2, 260.
27 M. Tress, E. U. Mapesa, W. Kossack, W. K. Kipnusu, M. Reiche and F. Kremer, Science, 2013, 341, 1371-1374.

28 D. Cangialosi, V. M. Boucher, A. Alegria and J. Colmenero, J. Chem. Phys., 2011, 135, 014901.

29 D. Cangialosi, V. M. Boucher, A. Alegria and J. Colmenero, Phys. Rev. Lett., 2013, 111, 095701.

30 V. M. Boucher, D. Cangialosi, A. Alegria and J. Colmenero, Thermochim. Acta, 2014, 575, 233-237.

31 S. Napolitano and D. Cangialosi, Macromolecules, 2013, 46, 8051-8053.

32 S. Napolitano, C. Rotella and M. Wübbenhorst, ACS Macro Lett., 2012, 1, 1189-1193.

33 C. Rotella, S. Napolitano, S. Vandendriessche, V. K. Valev, T. Verbiest, M. Larkowska, S. Kucharski and M. Wübbenhorst, Langmuir, 2011, 27, 13533-13538.

34 M. Erber, M. Tress, E. Mapesa, A. Serghei, K.-J. Eichhorn, B. Voit and F. Kremer, Macromolecules, 2010, 43, 7729-7733.

35 F. Kremer and A. Schönhals, Broadband Dielectric Spectroscopy, Springer Verlag, Berlin Heidelberg New York, 2002.

36 A. Serghei and F. Kremer, Rev. Sci. Instrum., 2008, 79, 026101. 37 K. S. Iyer, B. Zdyrko, H. Malz, J. Pionteck and I. Luzinov, Macromolecules, 2003, 36, 6519-6526.

38 D. Damiron, J. Mazzolini, F. Cousin, C. Boisson, F. D'Agosto and E. Drockenmuller, Poly. Chem., 2012, 3, 1838-1845.

39 B. Zdyrko, V. Klep and I. Luzinov, Langmuir, 2003, 19, 1017910187. 